\title{
Modulación del reflejo de sobresalto en población colombiana: evidencia de la interacción entre emoción y motivación*
}

\author{
Modulation of Startle Reflex in Colombian Population: Evidence \\ of the Interaction between Emotion and Motivation
}

Recibido: 2 de abril de 2014 | Revisado: 16 de septiembre de 2014 | Aceptado: 19 de octubre de 2014

\author{
CARlos Gantiva** \\ Universidad de San Buenaventura, Bogotá, Colombia \\ PEDRO GUERRA \\ JAIME VILA \\ Universidad de Granada, Granada, España
}

doi: 10.11144/Javeriana.upsy14-1.mrsp

Para citar este artículo: Gantiva, C., Guerra, P., \& Vila, J. (2015). Modulación del reflejo de sobresalto en población colombiana: evidencia de la interacción entre emoción y motivación. Universitas Psychologica, 14(1), 157-164. http://dx.doi.org/10.11144/ Javeriana.upsy14-1.mrsp

\footnotetext{
* Artículo original resultado de investigación

***Correos electrónicos: cgantiva@gmail.com,pguerra@ugr.es,jvila@ugr.es
}

\section{RES UMEN}

El objetivo de esta investigación fue comprobar el fenómeno de priming motivacional, observado a través de la modulación del reflejo de sobresalto en población colombiana. Participaron 73 estudiantes universitarios (38 hombres y 35 mujeres), los cuales fueron expuestos a 21 imágenes del Sistema Internacional de Imágenes Afectivas y a un estímulo sonoro de sobresalto de $105 \mathrm{~dB}$. Se midió la electromiografía del músculo orbicular del ojo para evaluar la magnitud del reflejo de sobresalto. A través de un ANOVA de medidas repetidas, se encontró que la valencia de las imágenes modula la magnitud del reflejo de sobresalto $\left(p<0.0001, \eta_{p}{ }^{2}=0.41\right)$ independientemente del sexo de los participantes. Se concluye que el fenómeno de priming motivacional también se presenta en esta población y que la metodología de la modulación del reflejo de sobresalto puede ser utilizada de forma confiable en ella.

Palabras clave

emoción; motivación; sobresalto; valencia

\section{A B S T R A C T}

The objective of this research was to test the phenomenon of motivational priming observed through the modulation of the startle reflex in Colombian population, involved 73 university students (38 men and 35 women) who were exposed to 21 pictures of the International Affective Pictures System and a sound startle stimulus of $105 \mathrm{~dB}$. Electromyography of the orbicularis oculi of the eye was measured to assess the magnitude of the startle reflex. Through repeated measures ANOVA we found that the pictures valence modulates the magnitude of the startle reflex $\left(p<0.0001, \eta_{p}{ }^{2}=0.41\right)$ and that modulation does not depend on the sex of the participants. It is concluded that motivational priming phenomenon occurs also in this population and this methodology can be used reliably in this population.

Keywords

emotion; motivation; startle; valence 


\section{Introducción}

Uno de los modelos con mayor soporte empírico para el estudio de las emociones es el modelo bioinformacional propuesto por Lang $(1995,2010)$. En él, las emociones se entienden como estados de predisposición a la acción, que se dan a partir de la activación de uno de los dos sistemas motivacionales primarios (el apetitivo y el defensivo), frente a la percepción de un estímulo relevante para el individuo (Lang, 2010; Lang \& Bradley, 2013).

Desde este modelo, las emociones tienen manifestaciones fisiológicas, conductuales y cognitivas que se reflejan en tres niveles diferentes. En el nivel 1 , se encuentran las respuestas conductuales y fisiológicas que dependen específicamente del contexto y que tienen su origen en la evolución filogenética, por ejemplo, la respuesta de lucha y escape, el incremento o decremento de la frecuencia cardiaca y el aumento en la conductancia galvánica de la piel, entre otras. Los estudios con mamíferos y reptiles muestran algunas de estas respuestas específicas ante situaciones amenazantes, por ejemplo, la respuesta de congelamiento, respuestas vocales, cambios en el tamaño y la forma (Blanchard \& Blanchard, 1989) y la desaceleración de la frecuencia cardiaca ante la percepción de una amenaza por la presencia de un predador, lo que se asocia a un incremento de la atención para aumentar el procesamiento de información y la elección de una acción adaptativa (Campbell, Wood, \& McBride, 1997). En el nivel 2, se agrupan los patrones específicos de respuesta del nivel anterior y dan origen a las emociones, por ejemplo, el miedo, la ira o la sorpresa, en las cuales se identifican ciertas estereotipias de respuesta ante diferentes contextos. Finalmente, en el nivel 3, se encuentran las dimensiones (valencia, arousal y dominancia), las cuales son compartidas por todas las emociones y sobre ellas se organiza la experiencia emocional (Lang, 1995, 2010).

La valencia es la dimensión a partir de la cual se estructura la emoción y es el componente motivacional de las emociones. La valencia depende del sistema motivacional activado, el apetitivo que se asocia con conductas consumatorias y de aproximación, y el defensivo que se relaciona con conductas de protección, escape y evitación (Lang $\&$ Davis, 2006). La valencia posee un sustrato neurofisiológico separado, lo que le otorga un carácter principal sobre las otras dos dimensiones (LeDoux, 2000); esto se ha observado en primates (Dolin, Zborovskaya, \& Zamakhovev, 1965), humanos y demás mamíferos, a través de registros de electroencefalografía e imágenes de resonancia magnética funcional (fMRI) (Bradley, 2009; Lang \& Bradley, 2010)

El arousal es la intensidad de la respuesta emocional; hace referencia a la cantidad de energía invertida en la emoción. No posee un sustrato neurofisiológico separado, sino que refleja la intensidad de la emoción producto de la activación del sistema motivacional apetitivo o defensivo (Lang, Davis, \& Öhman, 2000).

Finalmente, la dominancia es la dimensión evolutivamente más reciente; se refiere al control percibido sobre la emoción e implica la interrupción o continuidad de la respuesta conductual de la emoción (Cobos, García, Ríus, \& Vila, 2002). Estudios recientes con $\mathrm{fMRI}$ han demostrado que la dominancia activa regiones paralímbicas, incluida la ínsula anterior bilateral, ante imágenes con alta dominancia y la precúnea derecha, ante estímulos con baja dominancia (Jerram, Lee, Negreira, \& Gansler, 2014). La dominancia se asocia con funciones como la inhibición, el retraso, la evaluación del contexto y la planificación (Lang \& Bradley, 2010; Vila et al., 2001).

En este modelo, el uso de medidas psicofisiológicas para el estudio directo de la emoción, sin acudir a medidas subjetivas de autorreporte, ha sido ampliamente documentado, entre ellas el reflejo de sobresalto (Bianchin \& Angrilli, 2012; Bradley, Codispoti, Cuthbert, \& Lang, 2001; Cobos et al., 2002; Leite et al., 2012). Este reflejo se presenta en la mayoría de mamíferos y consiste en una respuesta esquelética muscular extensora-flexora que está bajo poco o ningún control voluntario, que recorre el cuerpo de arriba hacia abajo y se produce después de la percepción de un estímulo intenso e inesperado. Esta reacción es un reflejo defensivo que facilita el escape y la protección de los organismos y está compuesta 
por un patrón complejo de respuestas, entre ellas el parpadeo, que es la respuesta más rápida, confiable y estable (Bradley \& Lang, 2007). Se ha observado en estudios con animales que el reflejo de sobresalto se potencia ante situaciones amenazantes; este fenómeno fue reportado inicialmente por Brown, Kalish y Farber (1951), en ratas que fueron expuestas a un sonido intenso en presencia de un estímulo visual asociado previamente con una descarga eléctrica; posteriormente, se encontraron resultados similares en humanos (Hamm, Greenwald, Bradley, \& Lang, 1993).

En los estudios de laboratorio y según las guías internacionales para experimentación en psicofisiología, se ha demostrado que el reflejo de sobresalto se inicia entre 30 y 50 milisegundos después de la presentación de un sonido abrupto e intenso (ruido blanco de $105 \mathrm{~dB}, 50 \mathrm{~ms}$ de duración y de tiempo de subida instantáneo) y su principal forma de medida es a través de la magnitud de la respuesta de parpadeo, utilizando la electromiografía (EMG) del músculo orbicular (Blumenthal et al., 2005).

El reflejo de sobresalto es una medida objetiva y confiable de la valencia; se ha demostrado que el reflejo se modula según exista o no correspondencia entre la valencia del estímulo que genera el reflejo (aversiva) y el estado emocional en el que se encuentre la persona, este fenómeno es denominado priming motivacional (Lang, 1995, 2010). De esta forma, los experimentos consisten, por lo general, en la presentación de imágenes que pueden producir diferentes estados afectivos (imágenes placenteras, aversivas y neutrales) y de forma simultánea se presenta un ruido que genera el reflejo de sobresalto. El resultado que se ha encontrado es que cuando se presentan imágenes aversivas el reflejo de sobresalto se potencia, ya que existe correspondencia entre la valencia aversiva del ruido y la valencia aversiva que produce la imagen, pero cuando la imagen es apetitiva el reflejo de sobresalto se inhibe, debido a que no existe correspondencia entre las valencias de los dos estímulos (el sonido y la imagen); de esta forma, la modulación del reflejo de sobresalto es una medida objetiva y confiable para evaluar la valencia de la respuesta emocional ante diferentes estímulos (Bradley et al., 2001; Bradley \& Lang, 2007).
En Colombia, no existen investigaciones que hayan estudiado este fenómeno emocional y motivacional, por lo cual el objetivo del presente trabajo fue replicar el estudio de Lang (1995), con el fin de comprobar el fenómeno de priming motivacional, observado a través de la modulación del reflejo de sobresalto ante la visualización de imágenes afectivas, en una muestra de estudiantes universitarios colombianos. Los resultados de esta investigación permitirán replicar el uso de esta metodología objetiva para el estudio de las emociones, en Colombia.

\section{Método}

\section{Participantes}

A través de un muestreo no probabilístico, participaron voluntariamente 73 estudiantes universitarios de psicología, educación, ingeniería y ciencias políticas, procedentes de diferentes regiones del país: 38 hombres (20-31 años $\mathrm{M}$ edad $=23.64, \mathrm{DE}$ $=3.09$ ) y 35 mujeres (20-31 años; $M$ edad $=22.84$, $D E=3)$, sin reporte de trauma craneoencefálico o trastorno neurológico. Ningún participante estaba bajo tratamiento psicológico ni farmacológico, y se excluyeron personas con déficit no corregidos de tipo auditivo o visual.

\section{Diseño}

Se utilizó un diseño factorial mixto $2 \times 3$; como variable intersujeto: sexo (hombre y mujer) y como variable intrasujeto: valencia (imágenes apetitivas, neutrales y aversivas).

\section{Estímulos y procedimiento}

Se utilizaron 21 imágenes ( 7 apetitivas, 7 neutrales y 7 aversivas), seleccionadas del Sistema Internacional de Imágenes Afectivas ${ }^{1}$ ([IAPS]; Lang, Bradley, $\&$ Cuthbert, 2008), a partir de los valores normati-

1 Los códigos de las imágenes del IAPS fueron: apetitivas: 4676, 4693, 4668, 4692, 4698, 4623, 4677; neutrales 7026, 7161, 7179, 7013, 7018, 7041, 7021; aversivas: 1525, 1932, 6315, 6520, 9163, $3213,2683$. 
vos de la población colombiana (Gantiva, Guerra, \& Vila, 2011). Las imágenes fueron presentadas durante 6 segundos en una pantalla plana de 19 pulgadas localizada a $60 \mathrm{~cm}$ del participante. Todas las imágenes fueron presentadas dos veces, en cuatro órdenes contrabalanceados de 42 imágenes cada uno. En cada bloque, las imágenes fueron presentadas en orden aleatorio, el intervalo entre ensayos variaba aleatoriamente entre 10 a 14 segundos. El estímulo sonoro utilizado para generar el sobresalto fue un ruido blanco de $50 \mathrm{~ms}$ de duración, de $105 \mathrm{~dB}$ y con tiempo de subida instantáneo, administrado entre los segundos 4 y 5.5 después del inicio de la imagen (Blumenthal et al., 2005). El sonido de sobresalto se presentó aleatoriamente en la mitad de los ensayos de cada categoría de imagen y en cuatro intervalos entre ensayos. La duración completa del experimento fue de 16 minutos y se realizó en horas de la mañana.

\section{Equipos y medición de la respuesta psicofisiológica}

Se utilizó un computador con el software LabChart (v7.3, ADInstruments, Australia) para registrar la adquisición del EMG y un computador con el software E-Prime, versión 2.0 (Psychology Software Tools, Pennsylvania, USA) para controlar la presentación de los estímulos visuales y auditivos; ambos computadores estaban sincronizados para asegurar la precisión de las mediciones. La respuesta de parpadeo se midió a través de dos electrodos de 4 $\mathrm{mm} \mathrm{Ag/AgCl} \mathrm{colocados} \mathrm{sobre} \mathrm{el} \mathrm{músculo} \mathrm{orbicular}$ del ojo izquierdo (Blumenthal et al., 2005).

La actividad electromiográfica se registró con un equipo PowerLab 26T (ADInstruments), con una tasa de muestreo de $1000 \mathrm{~Hz}$ y con un filtro de banda entre 10 a $500 \mathrm{~Hz}$, rectificada e integrada con una constante de tiempo de $20 \mathrm{~ms}$. La magnitud del parpadeo se define como el mayor incremento de la actividad electromiográfica del músculo orbicular ocurrida en el intervalo de tiempo entre 20 a 150 ms después del estímulo de sobresalto, posteriormente a este valor se le resta la media del EMG de los $25 \mathrm{~ms}$ previos al inicio del sonido de sobresalto (Blumenthal et al., 2005). Para corregir la varia- bilidad individual en la amplitud del parpadeo, la magnitud del reflejo se transforma a puntuaciones T en cada sujeto.

A partir de lo anterior el $2.7 \%$ de todos los ensayos fueron excluidos debido a inestabilidad en la línea de base ( $>2 \mathrm{DE}$ por encima de la media de la línea de base de cada sujeto) o por una respuesta excesiva (outlier $>3 \mathrm{DE}$ por encima de la media de la magnitud del sobresalto para cada categoría de imagen en cada sujeto) (Cui et al., 2012).

\section{Análisis estadístico}

Inicialmente, se analizaron las variables sociodemográficas de los participantes a través de las pruebas $t$ de Student para muestras independientes, ANOVA y chi-cuadrado. Para evaluar el efecto de la valencia de las imágenes sobre la magnitud del reflejo de sobresalto, así como las posibles diferencias entre hombres y mujeres, se utilizó un ANOVA mixta $2 \times 3$ de medidas repetidas, como factor intersujeto: sexo, como factor intrasujeto: valencia (apetitiva, neutral y aversiva) y como variable dependiente la magnitud del reflejo de sobresalto. El factor de corrección Greenhouse-Geisser se utilizó para corregir cualquier violación de la esfericidad en el factor de medidas repetidas. Los análisis post hoc de los valores de las medias se llevaron a cabo a través de la comparación de pares con el factor de corrección Bonferroni. Finalmente, se llevó a cabo un análisis de correlación entre la edad y la magnitud del reflejo de sobresalto para cada categoría de imagen. El nivel de significancia para todos los análisis fue 0.05 y el tamaño del efecto también se reporta $\left(\eta_{\mathrm{p}}{ }^{2}\right)$.

\section{Consideraciones éticas}

El estudio fue aprobado por el Comité de Ética de la Universidad de San Buenaventura, Bogotá y todos los participantes firmaron el consentimiento informado como requisito para participar en la investigación. El estímulo sonoro utilizado no genera ningún daño físico o psicológico (Blumenthal et al., 2005), y toda la información recolectada se almacenó de forma anónima y confidencial. 


\section{Resultados}

\section{Variables sociodemográficas}

Inicialmente se analizaron las variables sociodemográficas de los participantes; no se encontraron diferencias significativas en ninguna de las variables ni entre los grupos. Con respecto a la edad, no se hallaron diferencias significativas entre hombres y mujeres $\left(t_{(51)}=-0.95, p=0.34\right)$, tampoco hubo diferencias significativas en la edad entre los participantes que estudiaban diferentes carreras $(F$ ${ }_{(3,52)}=0.23, p=0.87, \eta_{\mathrm{p}}{ }^{2}=0.01$ ). El análisis de la distribución de hombres y mujeres según la carrera tampoco mostró diferencias significativas $\left(\chi^{2}{ }_{(3)}=\right.$ $0.91, p=0.82$.

\section{Reflejo de sobresalto}

El ANOVA reveló un efecto principal significativo para valencia $\left(F_{(2,102)}=35.65, p<0.0001, \eta_{p}{ }^{2}=\right.$ 0.41 ), lo que indica que la valencia de las imágenes modula la magnitud del reflejo de sobresalto. Las imágenes apetitivas inhibieron significativamente el reflejo de sobresalto en comparación con las imágenes neutrales y con las aversivas (ambas $p$ $<0.0001$ ), y las imágenes aversivas potenciaron el reflejo de sobresalto en comparación con las apetitivas $(p<0.0001)$ y con las neutrales $(p=0.005)$. $\mathrm{El}$ análisis de tendencias muestra que el efecto de la valencia sobre el reflejo de sobresalto es lineal y significativo $\left(F_{(1,51)}=54.02, p<0.0001, \eta_{p}^{2}=\right.$ 0.51) (ver Figura 1).

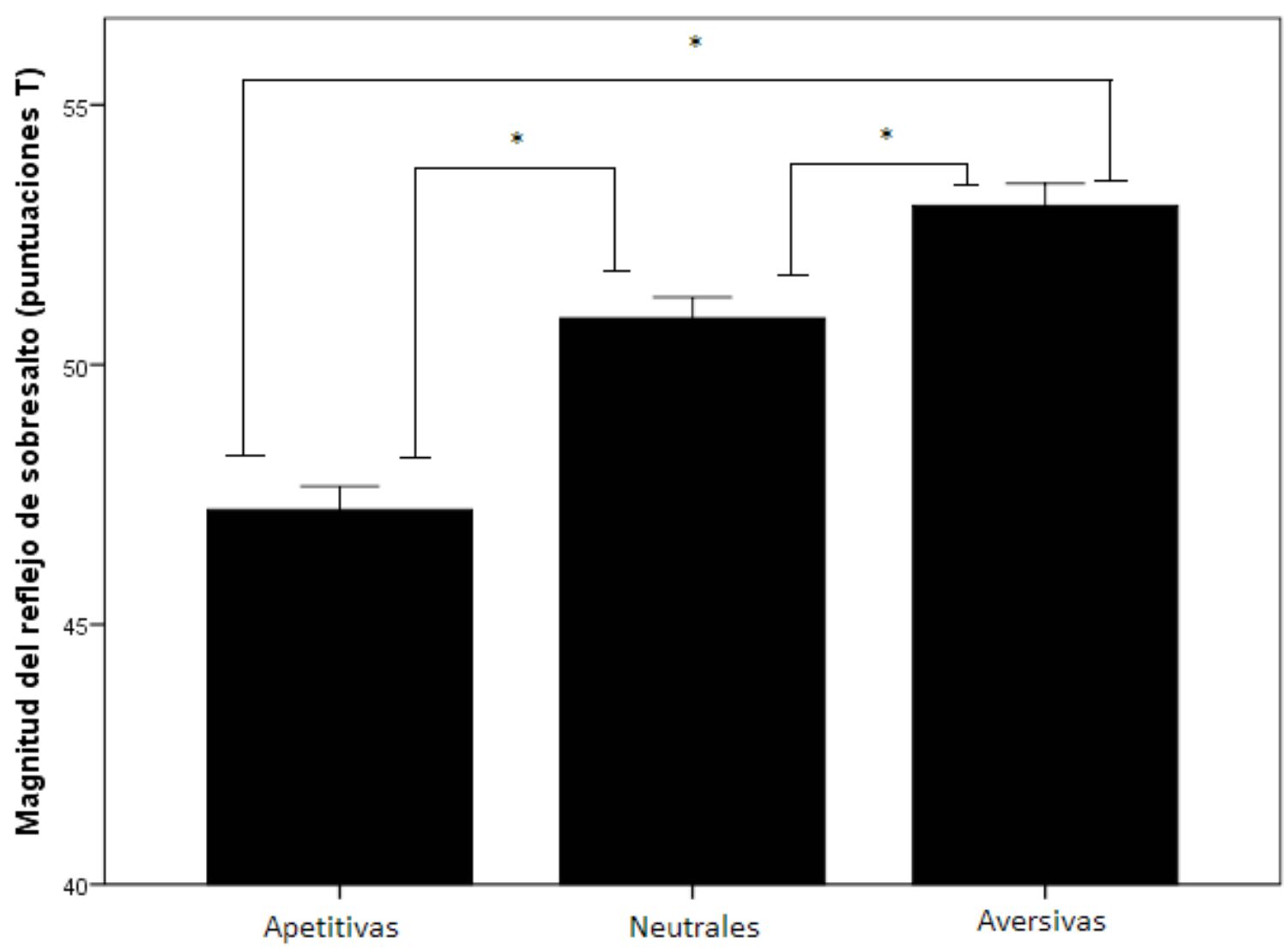

Figura 1. Modulación del reflejo de sobresalto según la valencia de las imágenes.

Nota. Las barras son el error estándar de la media. $* \mathrm{p}<0.01$

Fuente: elaboración propia 
Tabla 1

Estadísticos descriptivos de la magnitud del reflejo de sobresalto (puntuaciones T)

\begin{tabular}{lllllll}
\hline Valencia & Total & \multicolumn{3}{c}{ Hombres } & \multicolumn{3}{c}{ Mujeres } \\
\hline \multirow{3}{*}{ Apetitiva } & Media & DE & Media & DE & Media & DE \\
\cline { 2 - 7 } Neutral & 57.21 & 3.23 & 47.52 & 3.21 & 46.85 & 3.29 \\
Aversiva & 50.89 & 2.95 & 50.68 & 3.31 & 51.11 & 2.54 \\
\hline
\end{tabular}

Nota. DE: Desviación estándar.

Fuente: elaboración propia

El ANOVA no identificó un efecto principal significativo para sexo $(p=0.91)$ ni para la interacción Valencia $\times$ Sexo $(p=0.67)$, lo que indica que la modulación del reflejo de sobresalto no depende del sexo de los participantes (ver Tabla 1). Tampoco se encontró una relación significativa entre la edad de los participantes y la magnitud del reflejo de sobresalto para ninguna de las categorías de imágenes, apetitivas $\left(r_{(52)}=-0.25, p=0.07\right)$, neutrales $\left(r_{(52)}=0.06, p=0.62\right)$ y aversivas $\left(r_{(52)}\right.$ $=0.21, p=0.12$ ).

\section{Discusión}

La modulación del reflejo de sobresalto, a partir de la valencia afectiva de imágenes, es un fenómeno estudiado originalmente por Lang (1995), como estrategia metodológica para el estudio de la valencia, la dimensión más importante sobre la cual se estructura la experiencia emocional. Los resultados de estas investigaciones muestran que la valencia del estado emocional experimentada por la persona modula los reflejos defensivos, entre ellos el reflejo de sobresalto. Este fenómeno conocido como priming motivacional no solo se presenta en humanos, sino que fue identificado originalmente en ratas y en otros mamíferos (Brown et al., 1951).

El objetivo de esta investigación fue comprobar el fenómeno de priming motivacional en población colombiana a través de la modulación del reflejo de sobresalto ante la visualización de imágenes con diferente contenido afectivo. Los resultados muestran que la valencia de la imagen modula el reflejo de sobresalto; las imágenes apetitivas lo inhiben, las aversivas lo potencian y las neutrales generan un sobresalto intermedio. Estos mismos resultados han sido reportados en diferentes poblaciones y culturas (Bradley et al., 2001; Cobos et al., 2002; Grillon \& Baas, 2003; Muñoz, Idrissi, Sánchez-Barrera, Fernández-Santaella, \& Vila, 2013) lo que indica que el fenómeno de priming motivacional, por el cual los reflejos defensivos se modulan por la valencia del contexto, ocurre independiente de la cultura y puede ser utilizado de forma confiable y objetiva para el estudio de las emociones, en especial de la dimensión de valencia.

Los resultados también muestran que la modulación del reflejo de sobresalto se presenta de igual forma en hombres y en mujeres, sin diferencias, lo que indica que el fenómeno de priming motivacional no depende del sexo de los participantes. Este resultado es coherente también con los estudios desarrollados originalmente por Lang (1995) y por Bradley et al. (2001), lo que indica que la modulación de los reflejos defensivos tiene una base evolutiva y es un fenómeno adaptativo para la supervivencia, independiente de variables culturales e incluso del sexo.

Los resultados encontrados en población colombiana aportan evidencia empírica al modelo bio-informacional propuesto por Lang (2010), para la comprensión y el estudio de las emociones, debido a que se identificó nuevamente una fuerte influencia de la valencia sobre la magnitud del reflejo de sobresalto representada en el tamaño del efecto grande encontrado, lo que demuestra que la valencia aversiva de un estímulo potencia el reflejo de sobresalto y la valencia apetitiva lo inhibe; de esta forma, la modulación del reflejo de sobresalto se convierte en un indicador confiable de la valencia de la respuesta emocional; resultados similares han sido reportados en Norteamérica (Bradley et al., 2001; Lang, 1995) y España (Cobos et al., 2002). 
Adicionalmente, son múltiples los estudios con muestras específicas o clínicas que usan el reflejo de sobresalto como medida objetiva de la valencia (para una completa revisión consultar Filion, Dawson \& Schell, 1998; y Grillon \& Baas, 2003), por ejemplo, se ha observado un mayor incremento en la potenciación de los reflejos defensivos en personas que experimentan estados afectivos negativos como ira, tristeza, asco o miedo; en especial en sujetos con trastorno de estrés postraumático ante estímulos asociados al trauma (Grillon \& Baas, 2003), en personas con fobia específica ante estímulos asociados al tipo de fobia (Lang et al., 2000) y en personas con depresión (Taubitz, Robinson, \& Larson, 2013), entre otros. De igual forma, se ha observado la inhibición del reflejo de sobresalto en personas que experimentan estados afectivos positivos, por ejemplo en fumadores ante estímulos asociados al tabaco (Cui et al., 2012) y en consumidores excesivos de alcohol (Loeber et al., 2007), lo que indica la activación del sistema motivacional apetitivo ante estímulos asociados a la droga.

Finalmente, haber comprobado la modulación del reflejo de sobresalto en población colombiana permite desarrollar futuras investigaciones en Colombia para evaluar objetivamente la efectividad de las terapias clínicas. Por ejemplo, Kashdan, Adams, Read y Hawk (2012) demostraron que una hora de terapia de exposición logra disminuir la potenciación del reflejo de sobresalto en personas con fobia a las arañas. Este tipo de investigaciones permitirá aumentar la objetividad en las evaluaciones de la efectividad de las terapias psicológicas.

\section{Referencias}

Bianchin, M., \& Angrilli, A. (2012). Gender differences in emotional responses: A psychophysiological study. Physiology E Behavior, 105, 925-932. http:// dx.doi.org/10.1016/j.physbeh.2011.10.031

Blanchard, R. J., \& Blanchard, D. C. (1989). Attack and defense in rodents as ethoexperimental models for the study of emotion. Progressive NeuroPsychopharmacological E Biological Psychiatry, 13, 3-14.
Blumenthal, T., Cuthbert, B., Filion, D., Hackley, S., Lipp, O., \& van Boxtel, A. (2005). Committee report: Guidelines for human startle eyeblink electromyographic studies. Psychophysiology, 42(1), 1-15. doi: 10.1111/j.1469-8986.2005.00271.x

Bradley, M. (2009). Natural selective attention: Orienting and emotion. Psychophysiology, 46, 1-11. http:// dx.doi.org/10.1111/j.1469-8986.2008.00702.x

Bradley, M., Codispoti, M., Cuthbert, B., \& Lang, P. J. (2001). Emotion and motivation I: Defensive and appetitive reactions in picture processing. Emotion, 1(3), 276-298. http://dx.doi.org/10.1037//1528 3542.1.3.276

Bradley, M., \& Lang, P. (2007). Emotion and motivation. En Cacioppo, J., Tassinary, L., \& Berntson, G. (Eds.), The handbook of psychophysiology (pp. 602642). Nueva York: Cambridge University Press.

Brown, J. S., Kalish, H. I., \& Farber, I. E. (1951). Conditioned fear as revealed by magnitude of startle response to an auditory stimulus. Journal of Experimental Psychology, 32, 317-328.

Campbell, B. A., Wood, G., \& McBride, T. (1997). Origins of orienting and defensive responses: An evolutionary perspective. En P. J. Lang, R. F. Simons, \& M. T. Balaban (Eds.), Attention and orienting: Sensory and motivational processes (pp. 41-67). Hillsdale, NJ: Lawrence Erlbaum Associates.

Cobos, P., García, C., Ríus, F., \& Vila, J. (2002). Modulación emocional de la respuesta de sobresalto. Psicothema, 14(1), 106-111.

Cui, Y., Robinson, J., Versace, F., Lam, C., Minnix, J., Karam-Hage, M., ... Cinciripini, P. M. (2012). Differential cigarette-related startle cue reactivity among light, moderate, and heavy smokers. Addictive Behaviors, 37(8), 885-889. http://dx.doi. org/10.1016/j.addbeh.2012.02.003

Dolin, A. O., Zborovskaya, I. I., \& Zamakhovev, S. M. (1965). On the role of the orienting-exploratory reflex in conditioned reflex activity. En L. G. Voronin, A. N. Leontiev, A. R. Luria, E. N. Sokolov \& O. S. Vinogradova (Eds.), Orienting reflex and exploratory behavior (pp. 54-69). Washington, DC: American Institute of Biological Sciences.

Filion, D., Dawson, M., \& Schell, A. (1998). The psychological significance of human startle eyeblink mo- 
dification: A review. Biological Psychology, 47(1), $1-43$.

Gantiva, C., Guerra, P., \& Vila, J. (2011). Validación colombiana del sistema internacional de imágenes afectivas: evidencias del origen transcultural de la emoción. Acta Colombiana de Psicología, 14(2), 103-111.

Grillon, C., \& Baas, J. (2003). A review of the modulation of the startle reflex by affective states and its application in psychiatry. Clinical Neurophysiology, 114, 1557-1579. http://dx.doi.org/10.1016/S13882457(03)00202-5

Hamm, A. O., Greenwald, M. K., Bradley, M. M., \& Lang, P. J. (1993). Emotional learning, hedonic change, and the startle probe. Journal of Abnormal Psychology, 102(3), 453-465.

Jerram, M., Lee, A., Negreira, A., \& Gansler, D. (2014). The neural correlates of the dominance dimension of emotion. Psychiatry Research: Neuroimaging, 221, 135-141. doi: http://dx.doi.org/10.1016/j.pscychresns.2013.11.007

Kashdan, T., Adams, L., Read, J., \& Hawk, L. (2012). Can a one-hour session of exposure treatment modulate startle response and reduce spider fears? Psychiatry Research, 196, 79-82. http://dx.doi. org/10.1016/j.psychres.2011.12.002

Lang, P. J. (1995). The emotion probe: Studies of motivation and attention. American Psychologist, 50, 372-385.

Lang, P. J. (2010). Emotion and motivation: Toward consensus definitions and a common research purpose. Emotion Review, 2(3), 229-233. http:// dx.doi.org/10.1177/1754073910361984

Lang, P. J., \& Bradley, M. M. (2010). Emotion and the motivational brain. Biological Psychology, 84(3), 437-450.

Lang, P. J., \& Bradley, M. (2013). Appetitive and defensive motivation: Goal-directed or goal-determined? Emotion Review, 5(3), 230-234. http://dx.doi. org/10.1177/1754073913477511

Lang, P. J., Bradley, M., \& Cuthbert, B. (2008). International affective picture system (IAPS): Affective ratings of pictures and instruction manual (Technical Report A-8). Gainesville, FL: University of Florida.
Lang, P. J., \& Davis, M. (2006). Emotion, motivation, and the brain: Reflex foundations in animal and human research. Progress in Brain Research, 156, 3-29. http://dx.doi.org/10.1016/S0079. 6123(06)56001-7

Lang, P. J., Davis, M., \& Öhman, A. (2000). Fear and anxiety: Animal models and human psychophysiology. Journal of Affective Disorders, 61, 137-159.

LeDoux, J. (2000). Cognitive-emotional interaction: Listen to the brain. En R. D. Lane \& L. Nadel (Eds.), Cognitive neuroscience of emotion (pp. 129. 155). Nueva York: Oxford University Press.

Leite, J., Carvalho, S., Galdo-Alvarez, S., Alves, J., Sampaio, A., \& Gonçalves, Ó. (2012). Affective picture modulation: Valence, arousal, attention allocation and motivational significance. International Journal of Psychophysiology, 83, 375-381. http://dx.doi. org/10.1016/j.jpssycho.2011.12.005

Loeber, S., Croissant, B., Nakovics, H., Zimmer, A., Georgi, A., Klein, S., ... Flor, H. (2007). The startle reflex in alcohol-dependent patients: Changes after cognitive-behavioral therapy and predictive validity for drinking behavior. Psychotherapy and Psychosomatics, 76(6), 385-390. http://dx.doi. org/10.1159/000107567

Muñoz, M., Idrissi, S., Sánchez-Barrera, M., FernándezSantaella, M., \& Vila, J. (2013). Tobacco craving and eyeblink startle modulation using 3D immersive environments: A pilot study. Psychology of Addictive Behaviors, 27(1), 243-248. http://dx.doi. org/10.1037/a0028745

Taubitz, L., Robinson, J., \& Larson, C. (2013). Modulation of the startle reflex across time by unpleasant pictures distinguishes dysphoric from nondysphoric women. International Journal of Psychophysiology, 87, 124-129. http://dx.doi.org/10.1016/j. ijpsycho.2012.11.002

Vila, J., Sánchez, M., Ramírez, I., Fernández, M., Cobos, P., Rodríguez, S., ... Poy, R. (2001). El sistema internacional de imágenes afectivas (IAPS): adaptación española (Segunda parte). Revista de Psicología General y Aplicada, 54(4), 635-657. 